\title{
PENSAMIENTO REFLEXIVO: UNA APROXIMACIÓN INICIAL EN EL ÁMBITO DE LA FORMACIÓN DE FONOAUDIÓLOGOS
}

\section{REFLEXIVE THOUGHT: AN INITIAL APPROACH FOR THE TRAINING OF STUDENTS ENROLLED IN SPEECH AND HEARING SCIENCES PROGRAMMES}

\author{
Luis Romero R. \\ Profesor Asistente, Escuela de Fonoaudiología, Universidad de Chile. \\ Candidato a Doctor del Programa de Doctorado en Ciencias de la Educación, Facultad de Educación, \\ Pontificia Universidad Católica de Chile. \\ Correspondencia a: Iromero@med.uchile.cl
}

\begin{abstract}
RESUMEN
El presente trabajo reviste un esfuerzo particular por iniciar el tema del "pensamiento reflexivo en Fonoaudiólogos formadores de Fonoaudiólogos". Se revisan los conceptos de "pensamiento reflexivo" y "práctica reflexiva" así como también marcos teóricos con un sistema de clasificación de niveles de reflexión de racionalidad técnica, práctica y crítica. Se propone además, la aplicación en el ámbito fonoaudiológico de un tipo de capacitación de desarrollo de la práctica reflexiva.
\end{abstract}

Palabras Clave: Pensamiento reflexivo, práctica reflexiva.

\begin{abstract}
The present manuscript represents a specific effort on the commencement of "reflexive thought among speech pathologists who are involved in teaching students enrolled in Speech and Hearing Sciences Programmes". The concepts of "reflexive thought" and "reflexive practice" as well as a literature review with a system of classification of levels of reflexion of technical, practical and critical rationality are addressed. Also, the utilisation, within the field of speech and hearing sciences, of a type of training on the development of the reflexive practice is proposed.
\end{abstract}

Key words:. Reflexive thought, reflexive practice. 


\section{INTRODUCCIÓN}

El presente trabajo reviste un esfuerzo particular por iniciar el tema del "pensamiento reflexivo en Fonoaudiólogos formadores de Fonoaudiólogos". Una vez revisada la extensa literatura que existe hoy en día respecto del tema del pensamiento reflexivo en los diversos ámbitos, extraña la escasa literatura en la que se dé cuenta de la utilización de este proceso en la actividad docente de los Fonoaudiólogos. Éstos se desempeñan como formadores de personas, con el propósito que sean profesionales Fonoaudiólogos y que respondan a un perfil "ideal" en particular.

A la luz de la escasa literatura que existe en relación a la vinculación a la formación del Fonoaudiólogo con el pensamiento reflexivo es que se considera este artículo como una estrategia de "sensibilización y motivación" al cuerpo docente de las diversas escuelas de Fonoaudiología existentes hoy en nuestro país, para interiorizarse en el estudio y la práctica reflexiva.

Si bien es cierto, es lícito aseverar que el desarrollo de esta área de conocimiento se ha desarrollado principalmente en relación a la actividad de los Profesores en general, no es menos cierto que se puede y debe extender más allá de éstos, en consideración de la diversidad de profesionales que educan, (entre ellos el Fonoaudiólogo) y ver si para ellos, este enfoque resuelve algunos "problemas" que puedan estar tensionando lo vivido en su práctica formativa.

Inicialmente abordaremos algunas bases de definiciones en cuanto a lo que se entiende por pensamiento reflexivo, sus características, para luego sugerir relaciones para abordar el ámbito formativo del Fonoaudiólogo (desde la perspectiva realizada en la Escuela de Fonoaudiología de la Universidad de Chile) con el pensamiento y la práctica reflexiva.

Nuestra intención es tensionar esta práctica y de alguna forma dar cuenta de algunas propuestas de estrategias de reflexión en la formación de Fonoaudiólogos, sugerir también posibles puntos de vista desde los cuales proponer algunas directrices investigativas que aporten nueva información acerca de la formación de Fonoaudiólogos. 


\section{El pensamiento reflexivo}

Ligado al concepto de "práctica (o acción) reflexiva" y a la actividad del Profesor, se puede observar una gran diversidad de concepciones y enfoques acerca del "pensamiento reflexivo" de los profesores. Como un intento por construir una definición de lo que entenderemos por pensamiento reflexivo, tal vez debemos aproximarnos a través del apoyo que ofrecen términos relacionados. Uno de estos términos es el de práctica reflexiva. Ésta, supone actuar con un grado importante de originalidad, con cuidadosa examinación de los principios que sustentan dicha práctica. Supone también, una actitud casi permanente, estableciendo una relación analítica con la acción que realizamos ${ }^{1}$. Por el contrario, práctica irreflexiva implica un seguimiento o réplica de prácticas y principios no examinados ${ }^{2}$. Entonces ¿qué entenderemos por pensamiento reflexivo?

Como primer intento podríamos señalar, desde un punto de visto psicológico, que el pensamiento reflexivo es una consideración activa, persistente y cuidadosa de una creencia, a partir de los fundamentos que la sustentan y de las posteriores conclusiones hacia la cual tienden ${ }^{3}$.

Otra definición consiste en concebir al pensamiento reflexivo en la base de la práctica reflexiva. Se ha sostenido también que "la mejor manera de pensar", se denomina pensamiento reflexivo, es decir, el tipo de pensamiento que consiste en "darle vueltas a un tema en la cabeza y tomárselo en serio con todas sus consecuencias". Este tipo de pensamiento tendría 2 fases, por una parte implica: "a) un estado de duda, de vacilación, de perplejidad, de dificultad mental, en la que se origina el pensamiento; b) un acto de busca, de caza, de investigación, para encontrar algún material que esclarezca la duda, que disipe la perplejidad. La naturaleza del problema determina la finalidad del pensamiento y la finalidad controla el proceso de pensar"4.

En otras palabras, el pensamiento reflexivo sería aquella actividad cognitiva que sustenta el actuar con un grado importante de originalidad, a través de la cual se realiza una cuidadosa examinación de los principios que sustentan la práctica reflexiva. Este pensamiento reflexivo además, permite asumir una actitud casi permanente, estableciendo una relación 
analítica con la acción que se realiza. Implicaría los fundamentos de una postura, una forma de identidad o un hábitusi.

\section{Niveles de reflexión}

Estos conceptos, más o menos precisados, se han desarrollado ligados a tipos de discurso o "racionalidades" (Van Manen en Zeichner y Liston ${ }^{5}$ ).

El primer nivel de reflexión es el de racionalidad técnica. La preocupación dominante tiene que ver con la aplicación eficiente y eficaz del conocimiento educativo, para alcanzar unos fines dados. En este nivel ni los fines ni los contextos institucionales de aula, escuela, comunidad y sociedad se tratan como problemáticos" (Van Manen en Zeichner y Liston ${ }^{5}$.

Una visión similar de este nivel es presentado algunos años después (Schön, en Cornejo ${ }^{2}$ ) y se agregaba para este nivel la formación inicial y continua para los profesores. En este tipo de prácticas, el "dispositivo"ii implicaba a los profesores, someterse a experiencias estructuradas en laboratorios, luego de las cuales se evaluaba y discutía la actividad. La reflexión está hecha en base a los logros de los aprendices respecto de los objetivos de la clase. En definitiva se provee, a través de esta racionalidad, un ejercicio de información necesario para pulir sus destrezas en desarrollo². La preocupación dominante tiene que ver con la aplicación eficiente y eficaz del conocimiento educativo para unos fines determinados (Van Manen en Zeichner y Liston ${ }^{5}$.

El segundo nivel de reflexión es la de racionalidad práctica (Van Manen en Zeichner y Liston ${ }^{5}$ ) "este nivel se basa en una concepción de la acción práctica por la cual el problema reside en explicar y clarificar las suposiciones y predisposiciones que subyacen en los asuntos prácticos y juzgar las consecuencias educativas que conlleva una acción" (Van Manen en Zeichner y Liston 5 ). Un docente con estas características es quien puede pensar mientras está actuando. De esta manera puede responder a las situaciones que enfrenta sin importar la incertidumbre que estas situaciones impliquen, con originalidad y ponderando la conflictividad

\footnotetext{
i Habitus: Entendido como un conjunto de esquemas que permite infinidad de prácticas adaptativas a situaciones siempre renovadas, sin constituirse jamás en principios explícitos.

ii Dispositivo: Conjunto organizado de procedimientos con objetivos, procedimientos y proceso de evaluación.
} 
de las mismas (Schön, en Cornejo²). Se considera que toda acción va unida a unos compromisos de valor particular y que el que realiza la acción considera el valor de fines educativos rivales (Van Manen en Zeichner y Liston ${ }^{5}$ ) (Van Manen, op. cit. pp. 507).

El tercer nivel de reflexión lo constituye el de racionalidad crítica. Este nivel implica a los dos anteriores y además, "incorpora criterios morales y éticos dentro del discurso sobre la acción práctica. En este nivel, las preguntas centrales cuestionan qué objetivos educativos, experiencias y actividades conducen a formas de vida incluidas por el interés a la justicia, la equidad y si las disposiciones actuales satisfacen estas necesidades y propósitos especiales" (Van Manen en Zeichner y Liston ${ }^{5}$ ) (Van Manen, op. cit. pp. 507).

En este punto de la reflexión, se deben alcanzar cuestiones morales y éticas, indagar y reflexionar acerca de valores como la justicia, la equidad. Cuestionar lo ya determinado 0 establecido. Este nivel implica una necesaria reflexión acerca del "deber ser" y no la aceptación de las situaciones "como ellas son". (Van Manen en Zeichner y Liston ${ }^{5}$ ) (Van Manen, op. cit.)

\section{El accionar reflexivo en la formación de Fonoaudiólogos}

En el ámbito de la formación de Fonoaudiólogos el pensamiento reflexivo puede ser una herramienta para tomar conciencia acerca de la propia situación. De esta forma, considerar las posibilidades para actuar sobre lo que se considera decente, humano y justo en la formación de cualquier profesional y de manera especial con aquéllos cuyo trabajo implica interactuar con personas que presentan alteraciones en la comunicación.

La utilización del pensamiento reflexivo en la formación de Fonoaudiólogos implicaría entre otras cosas reflexionar en los tres niveles antes señalados. Es decir, reflexionar en el nivel técnico, práctico y crítico. No esté de más señalar que los primeros en utilizar la práctica reflexiva debieran ser los Fonoaudiólogos formadores de Fonoaudiólogos, de tal manera que con su práctica docente, faciliten a sus alumnos la reflexión técnica en su propia actividad formativa. 
Reflexionando en el nivel de racionalidad técnica, se debiera abordar principalmente, la forma de aplicar en forma eficaz y eficiente el conocimiento educativo para alcanzar así los fines curriculares que hablan de la adquisición de habilidades y destrezas por parte del alumno.

Reflexionando en el nivel de racionalidad práctica o praxiológica, vamos un poco más allá. En este nivel debiéramos buscar reflexivamente explicaciones y clarificaciones acerca de las suposiciones y predisposiciones que subyacen en la actividad práctica formadora del alumno, juzgar además, las consecuencias educativas que conllevarían dicha actividad práctica, cualquiera sea el área específica en la que se esté formando al futuro Fonoaudiólogo.

Reflexionar en el tercer nivel de racionalidad crítica, implica necesariamente, además de los niveles anteriormente señalados, incorporar dentro del quehacer y del pensar acerca del quehacer, criterios morales y éticos en el discurso sobre la acción práctica. Debiéramos permitirnos cuestionar qué objetivos educativos en la formación de Fonoaudiólogos, qué experiencias y actividades formativas, conducen a formas de vida influidas por el interés hacia valores como la justicia, la equidad, el bien común, dando así una clara dimensión político social a la formación del Fonoaudiólogo. Debiéramos reflexionar si las disposiciones actuales en la formación de formadores de Fonoaudiólogos y por lo tanto de los alumnos, satisfacen las necesidades que plantea la sociedad en la formación de Fonoaudiólogos y contribuyen a formar realmente a un profesional altamente capacitado (útil al mismo grupo al cual él pertenece), con alto grado de incidencia en el bienestar de la comunidad.

\section{Cómo alcanzar niveles de reflexión constructivo (técnico, praxiológico, crítico).}

El breve y tal vez superficial análisis presentado anteriormente, puede verse fortalecido si nos hacemos cargo también de las acciones estratégicas relacionadas con la enseñanza y formación de Fonoaudiólogos (docentes). Este punto es necesario destacarlo de manera especial, pues es tal vez una de las formas más propicias de llegar a los alumnos. Es clave implicar a los "formadores de Fonoaudiólogos", ya que cualquier estrategia que quiera ser eficaz requerirá que todo el cuerpo docente se "ponga en la onda". Se puede pensar en comenzar incluso por los más sensibilizados y que tengan mejores probabilidades de liderazgo 
e irradiación hacia sus demás colegas. Este enfoque entonces, es una propuesta de resolución de algunos "problemas" que puedan estar viviendo en su práctica formativa.

Una de las estrategias para formar en la práctica reflexiva tiene relación con concebir instancias de capacitación, más bien vistas como "empoderamiento" de los docentes Fonoaudiólogos; así abrir y crear espacios en las escuelas de Fonoaudiología que permitan plantear cuestiones de interés ${ }^{6}$. El empoderamiento se relaciona con el hacerse cargo, en propiedad de ciertas parcelas de nuestra vida a las que (eventualmente) se nos niega el acceso. Implica, por lo tanto, formas de vida que nos permitan dar un mayor sentido al medio en donde reflexionamos y actuamos ${ }^{6}$.

Al reflexionar en los tres niveles antes señalados, surge la necesidad de develar la naturaleza de las fuerzas que de alguna forma nos estaban determinando. Así, empezamos a buscar formas de perfeccionarnos. Una de estas formas de perfeccionamiento, se relaciona con recurrir a cuatro tipos de acciones ${ }^{\text {iii }}$ (que se corresponden con cuatro series de preguntas) ${ }^{6}$ (Smyth, op. cit.). Señaladas sucintamente, la primera acción es descripción que se relaciona con las preguntas ¿Qué es lo que hago? ¿Cuáles son mis prácticas? ; la segunda acción es la inspiración que se relaciona con las preguntas ¿Cuál es el sentido de la enseñanza que imparto? ¿Qué teorías expresan mis prácticas?; la tercera acción es confrontación que se relaciona con las preguntas ¿Cómo llegué a ser de esta forma? ¿Cuáles son las causas, supuestos, valores creencias? ¿De dónde proceden? Y finalmente, la cuarta acción que es la reconstrucción que se relaciona con las preguntas ¿Cómo podría hacer las cosas de otra manera? ¿Cómo podría cambiar? ¿Qué podría hacer diferente? ¿Qué es lo que tendría que hacer para introducir esos cambios? Responder a este cuestionamiento constituye una forma válida de desarrollar pensamiento reflexivo en torno a su práctica docente. Pero el docente Fonoaudiólogo también puede utilizar sus propias capacidades para formular e implementar programas de cambio, apoyados inicialmente, tal ves con estrategias como las aquí presentadas.

\footnotetext{
iii Acciones del proyecto de Perfeccionamiento Alternativo. John Smyth.
} 


\section{Síntesis}

Durante el proceso de pensamiento reflexivo acerca de las preguntas y de cómo plantearnos frente a las respuestas, los docentes Fonoaudiólogos podrían constituirse en agentes de cambio. Sea ésta tan sólo una propuesta inicial a modo de "sensibilización y motivación" a los docente formadores de Fonoaudiólogos de nuestro país, para luego interiorizarse en el estudio y la práctica reflexiva.

Si bien es cierto aquí se ha presentado una propuesta concreta de capacitación y empoderamiento de la práctica reflexiva susceptible de realizar por docentes Fonoaudiólogos, no debemos cegarnos a la posibilidad y necesidad de investigar en este ámbito. Ésta constituye otra forma de afrontar y subsanar la escasez de datos relacionados con la práctica reflexiva en Fonoaudiólogos en particular, y de profesionales de la salud en general.

\section{REFERENCIAS}

1. Perrenoud, P. (2004) Desarrollar la práctica reflexiva en el oficio de enseñar. Crítica y fundamentos, España: Graó.

2. Cornejo, J. (2003) El pensamiento reflexivo entre profesores. Pensamiento educativo. Vol. 32, pp. 343-373. Santiago de Chile.

3. Cazau, P.( 2004) Vocabulario Psicológico. http://galeon.hispavista.com/pcazau/vocps_pq.htm, Visitada 30 de noviembre de 2004 01:53 horas.

4. Dewey, J, (1989) Cómo pensamos, nueva exposición entre pensamiento y proceso educativo, Bs As: Paidós.

5. Zeichner, K. y Liston, D. (1999) Enseñar a reflexionar a los futuros docentes. En A. Pérez, J. Barquín y J.F. Angulo (eds.) Desarrollo profesional del docente. Política, investigación y práctica. (pp. 506-532), Madrid: Akal.

6. Smyth, J. (1991) Una pedagogía crítica de la práctica en el aula. Revista de Educación, № 294, (pp. 275-300). Madrid.

Recibido : : Agosto 2006

Aceptado : Abril 2007 